Western University

Scholarship@Western

Obstetrics \& Gynaecology Publications

Obstetrics \& Gynaecology Department

8-1-2005

p38 mitogen-activated protein kinase (MAPK) first regulates filamentous actin at the 8-16-cell stage during preimplantation development.

Andrew J M Paliga

David R Natale

Andrew J Watson

Follow this and additional works at: https://ir.lib.uwo.ca/obsgynpub

Part of the Obstetrics and Gynecology Commons

Citation of this paper:

Paliga, Andrew J M; Natale, David R; and Watson, Andrew J, "p38 mitogen-activated protein kinase (MAPK) first regulates filamentous actin at the 8-16-cell stage during preimplantation development." (2005). Obstetrics \& Gynaecology Publications. 45.

https://ir.lib.uwo.ca/obsgynpub/45 


\title{
p38 mitogen-activated protein kinase (MAPK) first regulates filamentous actin at the 8-16-cell stage during preimplantation development
}

\author{
Andrew J.M. Paliga* $† \ddagger$, David R. Natale ${ }^{*} \dagger^{1}$ and Andrew J. Watson ${ }^{*} \ddagger^{2}$ \\ *Department of Physiology and Pharmacology, University of Western Ontario, London, ON, Canada N6A 5C1, †Department of Obstetrics \\ and Gynaecology, University of Western Ontario, London, ON, Canada N6A 4G5, and ¥Child Health Research Institute, 5th Floor, \\ Victoria Research Laboratories, 800 Commissioners Road East, London, ON, Canada N6A 4G5
}

Background information. The MAPK (mitogen-activated protein kinase) superfamily of proteins consists of four separate signalling cascades: the c-Jun N-terminal kinase or stress-activated protein kinases (JNK/SAPK); the ERKs (extracellularsignal-regulated kinases); the ERK5 or big MAPK1; and the p38 MAPK group of protein kinases, all of which are highly conserved. To date, our studies have focused on defining the role of the p38 MAPK pathway during preimplantation development. p38 MAPK regulates actin filament formation through the downstream kinases MAPKAPK2/3 (MAPKactivated protein kinase 2/3) or MAPKAPK5 [PRAK (p38 regulated/activated kinase)] and subsequently through HSP25/27 (heat-shock protein $25 / 27$ ). We recently reported that 2 -cell-stage murine embryos treated with cytokine-suppressive antiinflammatory drugs (CSAID ${ }^{\mathrm{TM}}$; SB203580 and SB220025) display a reversible blockade of development at the 8-16-cell stage, indicating that p38 (MAPK) activity is required to complete murine preimplantation development. In the present study, we have investigated the stage-specific action and role of p38 MAPK in regulating filamentous actin during murine preimplantation development.

Results. Treatment of 8-cell-stage embryos with SB203580 and SB220025 (CSAID ${ }^{\text {TM}) ~ r e s u l t e d ~ i n ~ a ~ b l o c k a d e ~ o f ~ p r e-~}$ implantation development, loss of rhodamine phalloidin fluorescence, MK-p (phosphorylated MAPKAPK2/3), HSP-p (phosphorylated HSP25/27) and a redistribution of $\alpha$-catenin immunofluorescence by $12 \mathrm{~h}$ of treatment. In contrast, treatment of 2- and 4-cell-stage embryos with CSAID ${ }^{T M}$ drugs resulted in a loss of MK-p and HSP-p, but did not result in a loss of rhodamine phalloidin fluorescence. All these effects of p38 MAPK inhibition were reversed upon removal of the inhibitor, and development resumed in a delayed but normal manner to the blastocyst stage. Treatment of 8-cell embryos with PD098059 (ERK pathway inhibitor) did not affect development or fluorescence of MK-p, HSP-p or rhodamine phalloidin.

Conclusion. Murine preimplantation development becomes dependent on p38 MAPK at the 8-16-cell stage, which corresponds to the stage when p38 MAPK first regulates filamentous actin during early development.

\footnotetext{
${ }^{1}$ Present address: Health Sciences Centre, The University of Calgary, 3330 Hospital Drive NW, Calgary, AB, Canada T2N4N1.

${ }^{2}$ To whom correspondence should be addressed (email awatson@uwo.ca). Key words: blastocyst, cytoskeleton, embryo, in vitro fertilization.

Abbreviations used: DAPI, 4,6-diamidino-2-phenylindole; ERK, extracellularsignal-regulated kinase; hCG, human chorionic gonadotropin; HSP, heat-shock protein; HSP-p, phosphorylated HSP25/27; KSOMaa, potassium simplex optimized medium plus amino acid; MAPK, mitogen-activated protein kinase; MAPKAPK, MAPK-activated protein kinase; MEK, MAPK kinase/ERK kinase; MK-p, phosphorylated MAPKAPK2/3; PFA, paraformaldehyde; PRAK, p38 regulated/activated kinase.
}

\section{Introduction}

Preimplantation development is characterized by the differentiation of the first epithelium, the trophectoderm, and this occurs as the apolar cells of the cleavage-stage embryo polarize, undergo compaction and finally become a differentiated epithelium that mediates embryo attachment and invasion into the uterine wall (Watson, 1992; Watson et al., 
1992; Fleming et al., 1994; Watson and Barcroft, 2001; Schultz, 2002). This process, which defines preimplantation development, is unique to eutherian mammals and allows for an investigation of the mechanisms controlling the 'de novo' differentiation of an epithelium (Kidder and McLachlin, 1985; McLachlin and Kidder, 1986; Watson et al., 1992; Fleming et al., 1994; Watson and Barcroft, 2001).

Research conducted over the past three decades has established roles for the cytoskeleton, cell junctions (adherens junctions, tight junctions and desmosomes) and ion transporters in mediating trophectoderm differentiation. From this research, the cell biology of trophectoderm differentiation has become welldefined (Kidder and McLachlin, 1985; McLachlin and Kidder, 1986; Watson, 1992; Watson et al., 1992; Fleming et al., 1994; Watson and Barcroft, 2001). More recently, however, investigations have focused on how these gene products may be regulated and their actions co-ordinated within the blastomere during compaction and progression towards the trophectoderm cell type. For example, studies have implicated members of the protein kinase $C$ family, calmodulin and RhoGTPases in these events (Clayton et al., 1999; Pauken and Capco, 1999, 2000; Capco, 2001).

We are interested in defining the roles of MAPK (mitogen-activated protein kinase) pathways during preimplantation development. The MAPK superfamily of proteins consists of four separate signalling cascades: the c-Jun N-terminal kinase or stress-activated protein kinases (Woodgett et al., 1996; Whitmarsh and Davis, 2001); the ERKs (extracellular-signal-regulated kinases) (Boulton and Cobb, 1991; Pouyssegur et al., 2002); the ERK5 or big MAPK1 (Lee et al., 1995; Zhou et al., 1995); and the p38 MAPK group of protein kinases (Han et al., 1994), all of which are highly conserved in all eukaryotic systems (Kyriakis and Avruch, 2001). The MAPK proteins respond co-operatively to several stimuli, resulting in the mediation of cellular responses that include apoptosis, immune responses as well as growth and differentiation, cytoskeletal rearrangements and cell proliferation.

To date, our studies have focused on the p38 MAPK pathway (Natale et al., 2004). This MAPK subfamily includes four protein isoforms: $338 \alpha, \beta, \gamma$ and $\delta$, which regulate cellular processes such as inflammation, cytoskeleton rearrangements, as well as cell proliferation and apoptosis (Enslen et al., 2000; Ono and Han, 2000; Kyriakis and Avruch, 2001). p38 MAPK regulates actin filament formation first through MAPKAPK2/3 (MAPK-activated protein kinase 2/3) or MAPKAPK5 [(PRAK (p38 regulated/ activated kinase)] and subsequently through HSP25/ 27 (heat shock protein 25/27) (Lavoie et al., 1995; Guay et al., 1997; Huot et al., 1998; Dalle-Donne et al., 2001; Khurana and Dey, 2003). We have determined that all principal members of the p38 MAPK family are expressed throughout murine preimplantation development (Natale et al., 2004). More importantly, we discovered that inhibition of p38 MAPK activity at the 2-cell stage of murine preimplantation development with cytokine suppressive anti-inflammatory drugs (CSAID $^{\text {TM }}$; SB203580 and SB220025 active forms; SB202474 inactive form control) results in a blockade of development at the 8-16-cell stage that is fully reversible for at least a $48 \mathrm{~h}$ treatment period (Natale et al., 2004). This class of compounds specifically inhibits p38 MAPK $\alpha$ and $\beta$ isoforms (Cuenda et al., 1995; Badger et al., 1996; Jackson et al., 1998; Davidson and Morange, 2000; Davies et al., 2000; Cirillo et al., 2002; English and Cobb, 2002).

In the present study, we have investigated the embryo-stage-specific action of p38 MAPK by examining its role in regulating filamentous actin during preimplantation development. Our results demonstrate that inhibition of p38 MAPK activity does not affect filamentous actin at the 2- or 4-cell stage of development. However, at the 8-cell stage, effects on filamentous actin are observed within $12 \mathrm{~h}$ of treatment with p38 MAPK inhibitors $\left(\mathrm{CSAID}^{\mathrm{TM}}\right.$ drugs SB203580 and SB220025) and this is accompanied by a decline in MK-p (phosphorylated MAPKAPK2/3) and HSP-p (phosphorylated HSP25/27) and a redistribution of $\alpha$-catenin. All of these effects of p38 MAPK inhibition are reversed upon removal of the inhibitor, and development resumes in a delayed but normal manner to the blastocyst stage.

\section{Results}

Effects of p38 MAPK inhibitors on filamentous actin in 2- and 4-cell-stage embryos

In our first study (Natale et al., 2004), we determined that 2-cell-stage embryos can be treated with CSAID $^{\text {TM }}$ drugs (SB203580 and SB220025 active 


\section{Figure 1 I Effects of p38MAPK inhibitors on phospho-MAPKAPK2 (MK-p) and phospho-HSP25/27 (HSP-p) and filamentous actin in 2-cell and 4-cell CSAID $^{\text {TM }}$-treated embryos}

Two-cell embryos were collected and cultured for $12 \mathrm{~h}$ in either $\mathrm{KSOMaa}+0.01 \%$ DMSO (vehicle control), KSOMaa + $20 \mu \mathrm{M}$ SB202474 (drug control; A, E) or KSOMaa + $20 \mu \mathrm{M} \mathrm{SB} 220025$ (C, G). Four-cell embryos were treated for $12 \mathrm{~h}$ in either $\mathrm{KSOMaa}+0.01 \%$ DMSO (vehicle control), KSOMaa $+20 \mu \mathrm{M}$ SB202474 (drug control; B, F) or KSOMaa + $20 \mu \mathrm{M}$ SB220025 (D, H). Green represents MK-p and HSP-p fluorescence, red indicates rhodamine fluorescence and blue denotes DNA. Co-localization of HSP-p and filamentous actin appear as yellow. MK-p in 2- and 4-cell control embryos was predominantly confined to the interphase nucleus of each blastomere (A, B). In the SB220025 treatment group, we observed a complete loss of MK-p fluorescence in both 2- and 4-cell treated embryos by $12 \mathrm{~h}$ of treatment (C, D). HSP-p co-localized with F-actin in the cell cortex encircling the periphery of each blastomere within all 2- and 4-cell embryos in the control

MK-p
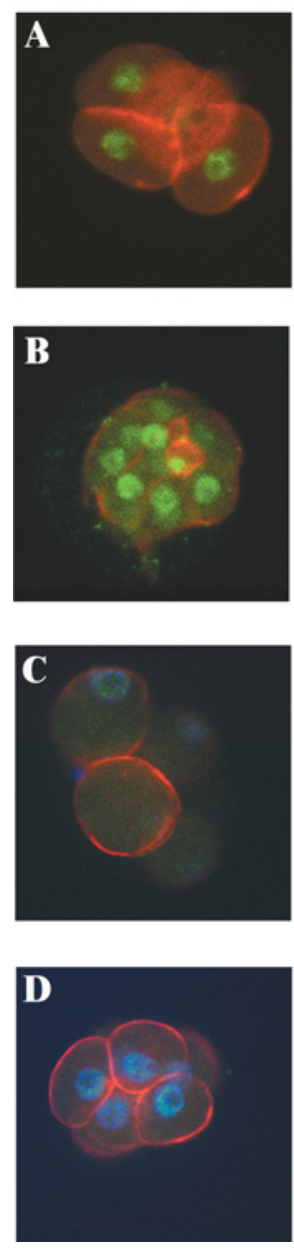

HSP-p
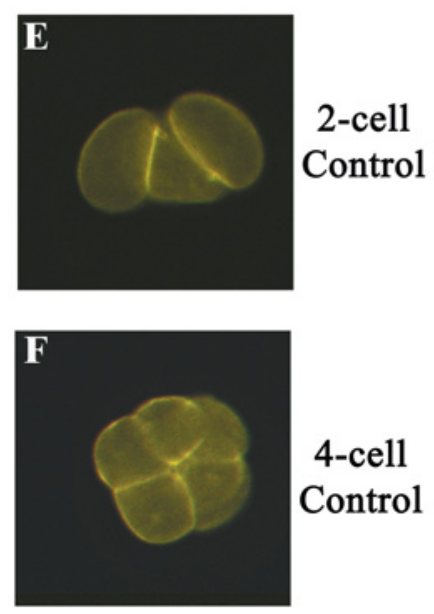

4-cell

Control

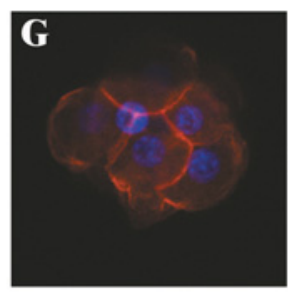

2-cell

SB220025

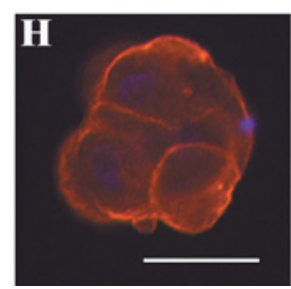

group (E, F). However, the SB220025-treated embryos displayed a complete loss of HSP-p fluorescence by $12 \mathrm{~h}(\mathbf{G}, \mathbf{H})$. In contrast with the loss of MK-p and HSP-p fluorescence, there was no reduction in cortical rhodamine fluorescence in SB220025-treated 2- and 4-cell-stage embryos compared with controls (A-H). Scale bar, $40 \mu \mathrm{m}$.

forms and SB202474 inactive form control) and still progress normally through the 4- and 8-cell stage only to arrest their development at the 8-16-cell stage. One possible reason for the stage-specific blockade of development at the 8-16-cell stage after CSAID $^{\mathrm{TM}}$ treatment is that the p38 MAPK pathway is not active in early cleavage-stage embryos. To investigate this possibility, in the present study, we investigated the stage-specific effects of CSAID $^{\mathrm{TM}}$ drugs on 2- and 4-cell-stage preimplantation embryos. Two-cell and 4-cell embryos were treated with $20 \mu \mathrm{M}$ SB220025 for $12 \mathrm{~h}$. MK-p in 2- and 4-cell control embryos was predominantly confined to the interphase nucleus of each blastomere (Figures $1 \mathrm{~A}$ and 1B). In the SB220025 treatment group, we observed a complete loss of MK-p fluorescence in both 2 - and 4-cell-treated embryos by $12 \mathrm{~h}$ of treatment (Figures 1C and 1D). HSP-p co-localized with F-actin in the cell cortex encircling the periphery of each blastomere within all 2- and 4-cell-stage embryos in the control group (Figures $1 \mathrm{E}$ and $1 \mathrm{~F}$ ). However, the SB220025-treated embryos displayed a complete loss of HSP-p fluorescence by $12 \mathrm{~h}$ (Figures $1 \mathrm{G}$ and $1 \mathrm{H}$ ). In contrast with the loss of MK-p and HSP-p fluorescence, there was no reduction in cortical rhodamine fluorescence in SB220025-treated 2- and 4-cell-stage embryos compared with controls (Figures $1 \mathrm{~A}-1 \mathrm{H}$ ).

\section{Effect of p38 MAPK inhibitors on the development of 8-cell embryos}

Since it was now apparent that the p38MAPK pathway was active during the 2- and 4-cell stages, we next wished to determine whether the absence of effect of CSAID ${ }^{\mathrm{TM}}$ treatment on development at these early stages was due to a requirement for a more extended CSAID ${ }^{\mathrm{TM}}$ treatment period or due to the onset of stage-specific sensitivity to CSAID ${ }^{\mathrm{TM}}$ treatment at the 8-cell stage. To distinguish between these possibilities, we focused subsequent experiments on the 8-cell stage of development. Treatment of 8-cell embryos with either SB203580 or SB220025 


\section{Figure 2 I Effect of p38 MAPK inhibitors on development of 8-cell embryos and morphology of embryos after $24 \mathrm{~h}$ CSAID $^{\text {TM }}$ treatment}

(A) Development of 8-cells stage embryos was evaluated after treatment in KSOMaa $+0.01 \%$ DMSO (vehicle control), $\mathrm{KSOMaa}+20 \mu \mathrm{M}$ SB202474 (drug control), KSOMaa $+20 \mu \mathrm{M} \mathrm{SB220025} \mathrm{or} \mathrm{KSOMaa+20 \mu M} \mathrm{SB203580.} \mathrm{a,} \mathrm{b,} \mathrm{significant}$ differences between percentage of embryos that reach the blastocyst stage; c, d, morula; and f, g, 8-cell-stage embryos. All differences were considered significant with $P \leqslant 0.05$. Development did not vary significantly between vehicle and drug controls. However, a significant reduction in the number of embryos that reached the blastocyst, morula and 8-cell stages was observed in both CSAID ${ }^{\mathrm{TM}}$ treatment groups. (B) (a) Vehicle control group; (b) KSOMaa $+20 \mu \mathrm{M} \mathrm{SB202474;} \mathrm{and} \mathrm{(c)} \mathrm{KSOMaa}+20 \mu \mathrm{M}$ SB220025. Eight-cell-stage embryos in both vehicle control (a) and drug control (b) groups display a high rate of development to the blastocyst stage, whereas 8-cell embryos treated with the active CSAID ${ }^{T M}$ (c) displayed a developmental blockade with the majority of treated embryos blocking at the $8-16$ cell stages. Scale bar $=100 \mu \mathrm{m}$.

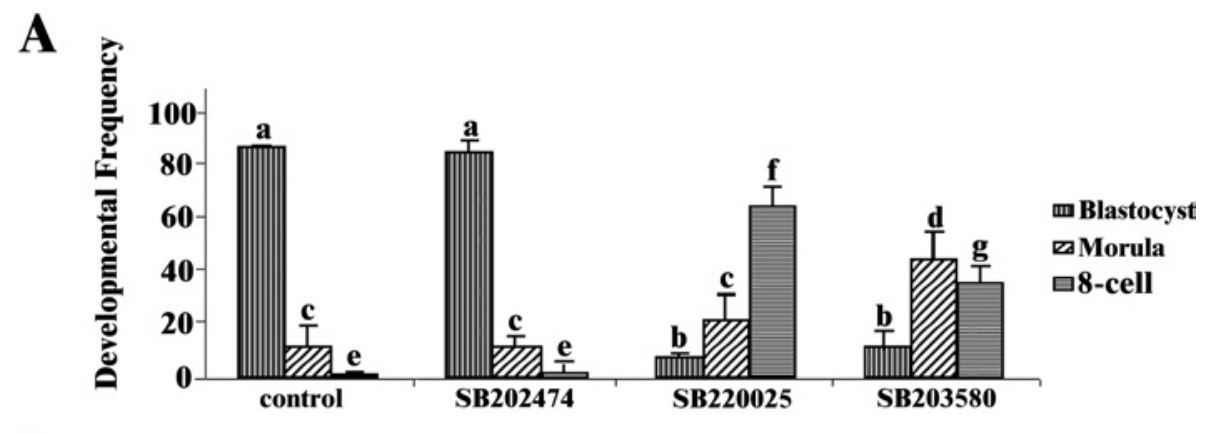

B
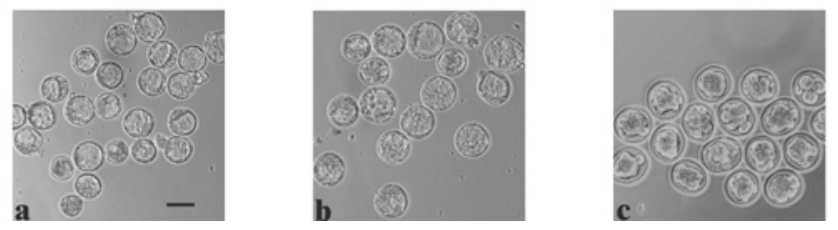

results in a significant decrease in the proportion of embryos that reached the blastocyst stage compared with controls (Figures $2 \mathrm{~A}$ and $2 \mathrm{~B}$ ). There was a corresponding significant increase in the number of embryos that remained at the morula (8-16 cell) stage in the SB203580 and SB220025 treatment groups compared with controls (Figures $2 \mathrm{~A}$ and 2B). After release from the drug treatments, all embryos recovered and developed to the blastocyst stage with the same frequency as controls after $24 \mathrm{~h}$ of recovery (results not shown). Cell viability was also assayed at this time using the vital dye, Trypan Blue (Humason, 1979), and there was no appreciable uptake of dye by embryos in any group (results not shown).

Time course of p38 MAPK inhibition on development of 8-cell embryos and the actin cytoskeleton

Since CSAID $^{\text {TM }}$ treatment at the 8-cell stage resulted in a more rapid onset of the developmental blockade than that observed when 2- or 4-cell-stage embryos were treated, we next defined a more precise time course for CSAID $^{\text {TM }}$ inhibition of p38 MAPK in 8-cell-stage embryos. Eight-cell embryos were treated with $20 \mu \mathrm{M}$ SB220025 and embryos were removed at 3, 6 and $12 \mathrm{~h}$ post-treatment for analysis. In both control groups (KSOMaa $+0.1 \%$ DMSO and KSOMaa + SB202474; where KSOMaa stands for potassium simplex optimized medium plus amino acids), MK-p was predominantly confined to the interphase nucleus of each blastomere, just as was observed for control 2- and 4-cell embryos (Figures $3 \mathrm{~A}$ and $3 \mathrm{~B}$ ). In the controls, for all time points, there was no change observed in either the localization or the intensity of MK-p. In the SB220025 treatment group, there was an obvious decrease in MK-p intensity by $3 \mathrm{~h}$, which was followed by a complete absence of MK-p fluorescence by $12 \mathrm{~h}$ of treatment (Figures 3C-3E). In contrast with the MK-p distribution, HSP-p co-localized with F-actin in the cell 


\section{Figure 3 I Time course of p38 MAPK inhibition on localization of MK-p, HSP-p, $\alpha$-catenin protein and the actin cytoskeleton in CSAID ${ }^{\mathrm{TM}}$-treated embryos}

8-cell embryos were cultured for up to $12 \mathrm{~h}$ in either $\mathrm{KSOMaa}+0.01 \%$ DMSO (vehicle control), KSOMaa + $20 \mu \mathrm{M}$ SB202474 (drug control) or KSOMaa + $20 \mu \mathrm{M}$ SB220025. A subset of embryos from each group was removed at 3, 6 and $12 \mathrm{~h}$, fixed in $2 \%$ PFA and then processed for whole-mount indirect immunofluorescence. Green represents MK-p protein localization. Co-localization of HSP-p and filamentous actin appear as yellow. Red indicates rhodamine phalloidin (A-O) and blue denotes DNA (A-O). No variation in localization pattern or intensity of MK-p, HSP-p or $\alpha$-catenin fluorescence was observed between vehicle and drug control groups (A, B, $\mathbf{F}, \mathbf{G}, \mathbf{K}, \mathbf{L})$. Localization also did not vary for these proteins in the drug-treated groups after $3 \mathrm{~h}$ of treatment $(\mathbf{C}, \mathbf{H}, \mathbf{M})$; however, a reduction in the levels of MK-p is apparent as indicated by the reduced nuclear fluorescence after $6 \mathrm{~h}$ of treatment (D). After $12 \mathrm{~h}$ of treatment, a further reduction in MK-p is observed combined with the complete loss of the rhodamine phalloidin fluorescence (E). A reduction in HSP-p fluorescence is observed at $6 \mathrm{~h}$ of treatment (I), with a complete loss of HSP-p and rhodamine phalloidin fluorescence by $12 \mathrm{~h}$ of treatment $(\mathbf{J})$. During $6 \mathrm{~h}$ of treatment, the distribution

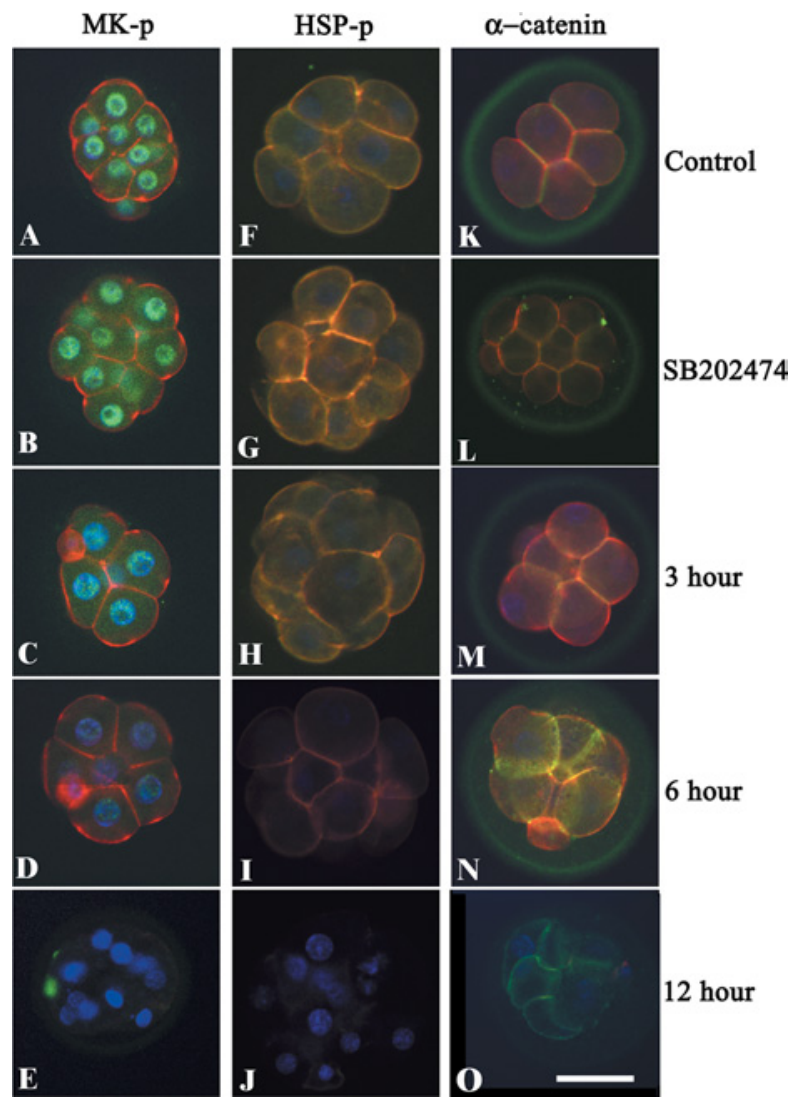

of $\alpha$-catenin fluorescence transitioned from a predominantly cortical pattern in adjacent cell-to-cell margins, to include a cytoplasmic fluorescent foci $(\mathbf{N})$. After $12 \mathrm{~h}$ of treatment, $\alpha$-catenin fluorescence intensity was considerably reduced by this treatment time (O). Scale bar $=50 \mu \mathrm{m}$.

cortex encircling the periphery of each blastomere within all embryos from both control groups (Figures $3 \mathrm{~F}$ and $3 \mathrm{G}$ ). However, the SB220025-treated groups of embryos displayed a reduction in HSP-p fluorescence by $6 \mathrm{~h}$, followed by a complete absence of fluorescence by $12 \mathrm{~h}$ (Figures $3 \mathrm{H}-3 \mathrm{~J}$ ). These experiments were repeated using $2 \mu \mathrm{M}$ SB220025 and identical results were obtained, with the exception that MK-p and HSP-p fluorescence was reduced to barely detectable levels, but never completely disappeared after treatment with this concentration of the inhibitor (results not shown).

\section{Effect of p38 inhibitors on F-actin in 8-cell-stage embryos}

Eight-cell control embryos displayed a cortical localization of $\mathrm{F}$-actin encircling the periphery of each blastomere (Figures 3A, 3B, 3F, 3G, 3K and $3 \mathrm{~L})$. There was no detectable change in actin localization or rhodamine phalloidin fluorescence intensity throughout the 3, 6 and 12 h experimental time points in the control groups (Figures $3 \mathrm{~A}, 3 \mathrm{~B}, 3 \mathrm{~F}$, $3 \mathrm{G}, 3 \mathrm{~K}$ and $3 \mathrm{~L}$ ). However, by $6 \mathrm{~h}$ of treatment with $20 \mu \mathrm{M}$ SB220025, an obvious reduction in the Factin fluorescence was observed, followed by a complete loss of rhodamine phalloidin fluorescence by the $12 \mathrm{~h}$ treatment time (Figures $3 \mathrm{C}-3 \mathrm{E}, 3 \mathrm{H}-3 \mathrm{~J}$ and $3 \mathrm{M}-3 \mathrm{O})$. These experiments were replicated using a $2 \mu \mathrm{M}$ concentration of SB220025 and identical outcomes were observed, except that the rhodamine phalloidin fluorescence was reduced to barely detectable levels, but did not completely disappear by the $12 \mathrm{~h}$ treatment time (results not shown).

\section{Effects of p38 MAPK inhibitors on adherens junction proteins in 8-cell-stage embryos}

Eight-cell control embryos displayed an expected fluorescence pattern that consisted predominantly of a cortical pattern confined to the juxtaposed borders of adjacent cells for both $\alpha$-catenin (Figures $3 \mathrm{~K}$ and 


\section{Figure 4 | Effect of MEK inhibition on development of murine embryos and localization of MK-p, HSP-p and phospho-p42/44 in PD098059- and SB220025-treated embryos}

(A) Murine embryos were flushed from timed-pregnant oviducts at the 2-cell stage (48 $\mathrm{h}$ post-hCG), pooled, washed and divided equally into four treatment groups consisting of (1) KSOMaa + 0.1\% DMSO, (2) KSOMaa + $1 \mu$ M PD098059 (PD98059), (3) KSOMaa + $10 \mu \mathrm{M}$ PD098059 and (4) KSOMaa + $100 \mu \mathrm{M}$ PD098059. Embryos were assessed for morphology and progression through to the blastocyst stage. No significant differences in the proportion of embryos developing to the blastocyst stage were observed between PD098058-treated and control groups. (B) Phospho-P42/44 fluorescence is represented in green (1 and 4), as is MK-p in panel 2. The yellow-orange fluorescence represents HSP-p protein co-localizing with rhodamine phalloidin (3). Red indicates rhodamine phalloidin, and blue denotes DNA (1-4). Panels 1-3 depict embryos from PD098059 treatments, whereas panel 4 displays embryos from the SB220025 treatment group. There was no difference in the fluorescence pattern of either MK-p or HSP-p in the PD098059-treated embryos (2, 3), although there was an absence of phospho-p42/44 fluorescence in these embryos (1). There was no variation in rhodamine phalloidin fluorescence in the PD098059-treated embryos (1-3). Similarly, no variation in the fluorescence of the phosphorylated-P42/44 antiserum was detected in the SB220025 treatment group (4). Rhodamine phalloidin fluorescence was reduced in the SB220025-treated embryos (4). Scale bar $=50 \mu \mathrm{m}$.

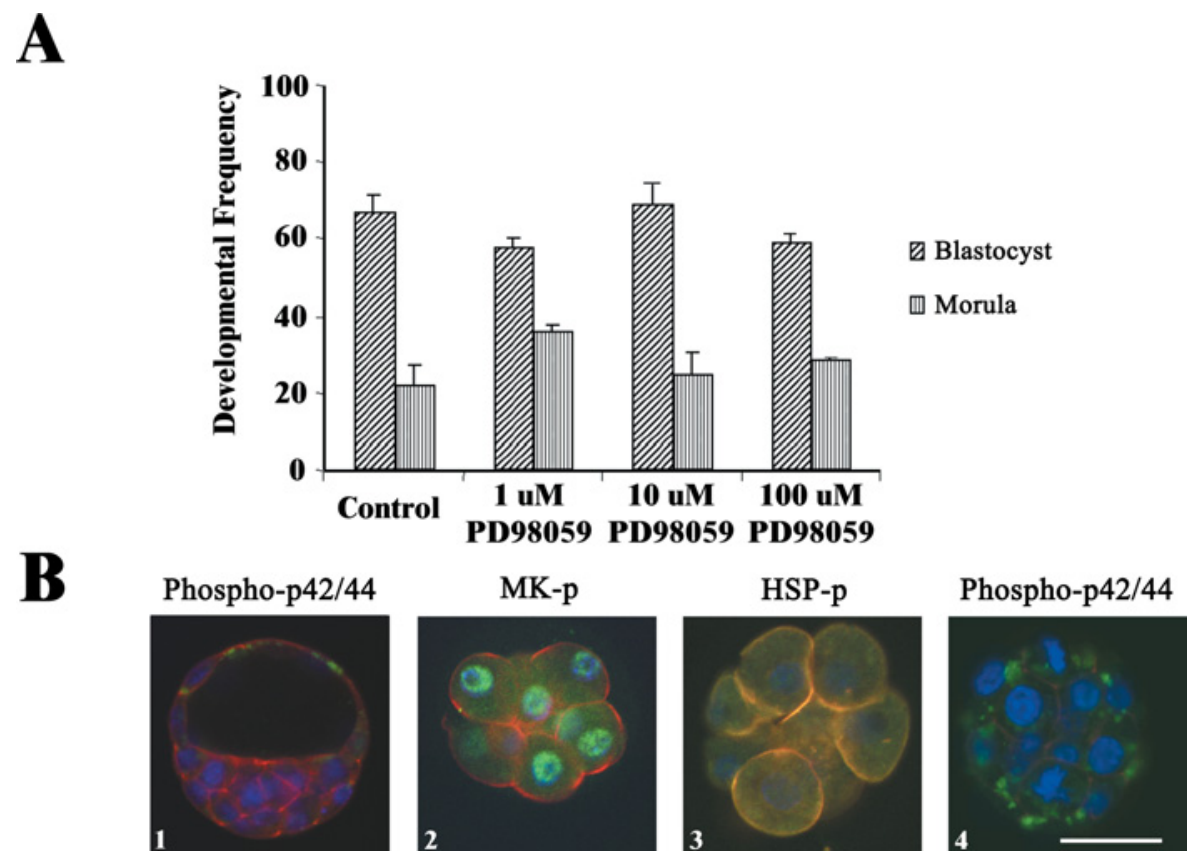

$3 \mathrm{~L})$ and $\beta$-catenin antisera (results not shown). After treatment with SB220025, $\beta$-catenin localization and intensity did not vary from that of controls at all observed time points (results not shown). In contrast, after $6 \mathrm{~h}$ of treatment with SB220025, the distribution of $\alpha$-catenin transitioned from a completely cortical pattern primarily confined to juxtaposed cell borders, to include an apolar localization (distinct fluorescence in free apical cell surfaces) as well as a cytoplasmic distribution consisting of diffuse fluorescent foci (Figure $3 \mathrm{~N}$ ). After $12 \mathrm{~h}$ of treatment, the $\alpha$-catenin fluorescence pattern was consistently reduced in intensity (Figure 3O).

\section{MEK-1 (MAPK/ERK kinase) inhibitor experiments}

To investigate the possible involvement of the ERK/ MAPK pathway in mediating these events, we conducted experiments with PD098059, which targets MEK-1 and thus is a potent inhibitor of ERK-1. Experiments employing this inhibitor did not affect development to the blastocyst stage (even at concentrations as high as $100 \mu \mathrm{M}$; Figure $4 \mathrm{~A}$ ) and did not affect the intensity or localization of MK-p or HSP-p fluorescence in the treated embryos [Figures $4 \mathrm{~B}(2)$ and 4B(3)]. PD098059 treatment was efficacious in these experiments as we observed a significant reduction in fluorescence of the phosphorylated 
form of $\mathrm{p} 42 / 44$ (p42/44-p; ERK-1/2), which is a downstream target of MEK-1 [Figure 4B(1)]. No effects on p42/44-p fluorescence were observed in embryos treated with the p38 MAPK inhibitor SB220025 [Figure 4B(4)].

\section{Embryo recovery from treatment with p38 MAPK inhibitors}

After $3 \mathrm{~h}$ of recovery in drug-free medium, SB220025-treated embryos displayed an abrupt reappearance of MK-p and HSP-p fluorescence (Figures 5D-5F). Once again, the MK-p fluorescence was predominantly confined to the interphase nuclei in each blastomere, whereas the HSP-p fluorescence was cortical, encircling the periphery of each cell (Figures 5D and 5E). MK-p was observed within the cytoplasm in dividing blastomeres (Figure 5D). Furthermore, $\alpha$-catenin fluorescence also recovered its intensity and exclusive basolateral localization pattern. No detection of a cytoplasmic $\alpha$-catenin was observed beyond $3 \mathrm{~h}$ of recovery (Figure $5 \mathrm{~F}$ ). Most importantly, the rhodamine phalloidin fluorescence reappeared by $3 \mathrm{~h}$ of recovery time, indicating that actin had reassembled to become filamentous, cortical actin once again (Figures 5D-5F). By $24 \mathrm{~h}$ of recovery time, the fluorescence patterns for MK-p, HSP-p, actin, $\alpha$-catenin and the appearance of blastocysts in the treated groups (Figures $5 \mathrm{G}-5 \mathrm{I}$ ) did not vary from the untreated controls (Figures 5A-5C).

\section{Discussion}

We have recently reported that mRNAs and polypeptides encoding p38 MAPK $\alpha, \beta, \gamma$ and $\delta$, and other members of the p38 MAPK signalling cascade including the downstream effectors, MK2, p38related PRAK (MK5) and HSP25/27, are expressed throughout murine preimplantation development (Natale et al., 2004). More importantly, we demonstrated that treatment of 2-cell-stage mouse embryos with p38 MAPK inhibitors SB203580 and SB220025 resulted in a reversible blockade of development that occurs at the 8-16-cell stage of development (Natale et al., 2004).

The present study investigated the stage-specific activation of p38 MAPK signalling, and has characterized the timing of p38 MAPK regulation of filamentous actin, $\alpha$-catenin and embryo development. We have demonstrated that treatment of 8-cell embryos with CSAID $^{\mathrm{TM}}$ drugs results in a reversible

\section{Figure 5 | Embryo recovery from treatment with p38 MAPK inhibitors}

Eight-cell embryos were collected and cultured for a period of $12 \mathrm{~h}$ in either KSOMaa $+0.01 \%$ DMSO (vehicle control), $\mathrm{KSOMaa}+20 \mu \mathrm{M} \mathrm{SB} 202474$ (drug control) or KSOMaa+ $20 \mu \mathrm{M}$ SB220025. After treatment, embryos were washed and returned to KSOMaa. Green represents MK-p and colocalization of HSP-p and filamentous actin appear as yellow. Red indicates rhodamine phalloidin (A-I) and blue denotes DNA (A-I). No variation in the distribution of these proteins was observed between the vehicle and drug control groups (A-C). MK-p fluorescence reappeared by $3 \mathrm{~h}$ of recovery and was confined to the nuclei except in cells undergoing mitosis (D). HSP-p fluorescence reappeared by $3 \mathrm{~h}$ (E). $\alpha$-Catenin fluorescence was also restored by $3 \mathrm{~h}$ of recovery time (F). Rhodamine phalloidin fluorescence recovered within $3 \mathrm{~h}$ (D-F). By $24 \mathrm{~h}$ of recovery, all CSAID ${ }^{\mathrm{TM}}$-treated groups displayed a normal developmental frequency to the blastocyst stage and a normal localization for MK-p (G), HSP-p (H), $\alpha$-catenin (I) and filamentous actin (G-I) that was indistinguishable from that of controls. Scale bar $=50 \mu \mathrm{m}$.

\section{Control} Recovery
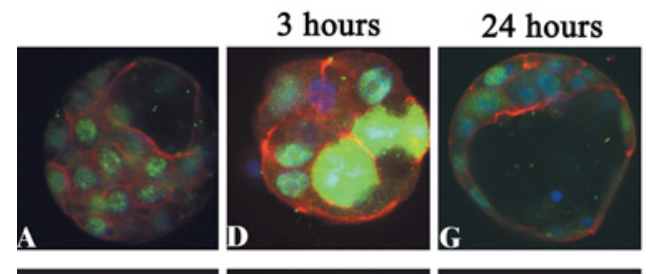

MK-p
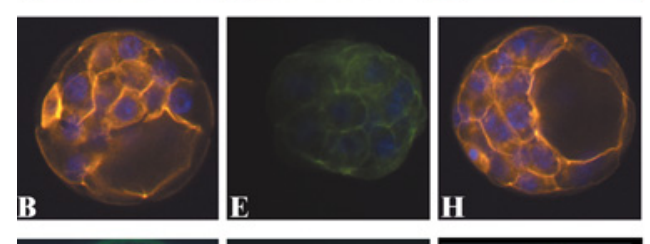

HSP-p
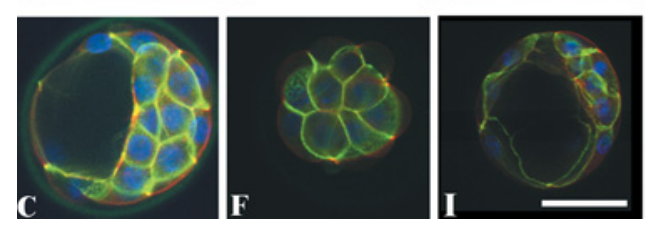

$\alpha$-catenin

blockade of development (by $12 \mathrm{~h}$ ) that is associated with a loss of phosphorylation of MK2, HSP25/27, redistribution of $\alpha$-catenin and a loss of filamentous actin. Furthermore, these effects of p38 MAPK inhibition on 8-16-cell-stage embryos are fully reversible and are not observed when the ERK pathway is inhibited. The most intriguing finding of our study is that despite the presence of an active p38 
MAPK pathway at the 2- and 4-cell stage of development, this pathway does not regulate filamentous actin in these embryo stages. Therefore the p38 MAPK pathway plays an important stage-specific role in mediating the events of preimplantation development. One of these roles is to promote the assembly of filamentous actin beginning at the 8-cell stage of development.

In several cell systems, p38 MAPKs regulate actin dynamics (Guay et al., 1997; Dalle-Donne et al., 2001; Khurana and Dey, 2003). The signalling cascade propagates actin filament restructuring through the phosphorylation of p38 MAPK. Active p38 MAPK in turn phosphorylates MAPKAPK2, which then activates HSP27 (Benndorf et al., 1994; DalleDonne et al., 2001; Kyriakis and Avruch, 2001). The present study demonstrated that CSAID ${ }^{\mathrm{TM}}$ inactivation of p38 MAPK results in a corresponding decrease in the levels of phospho-forms of MAPKAPK2, and HSP25/27, which precedes a significant decrease in filamentous actin in the 8-16-cell embryo. These observations concur with a recent model proposed by Gerthoffer and Gunst (2001), which predicts that p38 MAPK exerts its influences on actin by modulating PP2A (protein type 2 phosphatase), which in turn regulates the polymerization and stability of filamentous actin fibres. Future studies will be directed at testing the validity of this model during preimplantation development.

Other kinase pathways can cross-talk with the p38 MAPK pathway. For example, ERK can interplay with the p38 MAPK pathway at the level of MKK4 (MAPK kinase 4) (Kyriakis and Avruch, 2001). However, inhibition of 2-cell embryos with the MEK1 (ERK1) inhibitor PD098059 at concentrations of 1, 10 and $100 \mu \mathrm{M}$ did not affect development, as a normal progression to the blastocyst stage was observed in their presence. This was associated with no obvious effects on the actin cytoskeleton. For these reasons, we conclude that the ERK/MAPK pathway is not a principal regulator of the actin cytoskeleton during preimplantation development.

Actin plays an integral role in a significant number of cellular processes, one of which is the anchoring and maintenance of the adherens junctions during embryo compaction (Pratt et al., 1982). Adherens junctions are cadherin-dependent adhesive structures that are intricately linked to the actin microfilament network (Kidder and McLachlin, 1985; Levy et al., 1986;
McLachlin and Kidder, 1986; Ohsugi et al., 1996, 1997, 1999; Goval et al., 2000). In the presence of calcium, cadherin molecules of adjacent cells bind forming a junction that brings the cell membranes of neighbouring cells into close proximity. Cadherin, a membrane-spanning protein, is bound by $\beta$-catenin, which is in turn bound to $\alpha$-catenin. $\alpha$-Catenin then interacts with the filamentous actin network to anchor the junctional complex (Perez-Moreno et al., 2003). The assembly and formation of adheren junctions between neighbouring cells in the developing embryo results in the closer and more intimate association of cells, eventually obscuring the individual cell borders and resulting in the morphogenetic event of compaction (Kidder and McLachlin, 1985; Levy et al., 1986; McLachlin and Kidder, 1986; Ohsugi et al., 1996, 1997, 1999; Goval et al., 2000). We predict that the changes in $\alpha$-catenin localization that we observed after CSAID ${ }^{\mathrm{TM}}$ treatment occur as a consequence of depolymerization of the filamentous actin, which results in the formation of unstable adherens junctions in CSAID $^{\mathrm{TM}}$-treated embryos. This result is intriguing and provides a possible explanation for why most of the treated embryos do not complete compaction in the presence of CSAID ${ }^{\mathrm{TM}}$ drugs and block at the 8-16-cell stage. Without proper assembly of stable adherens junctions, compaction cannot proceed.

Recovery from CSAID ${ }^{\mathrm{TM}}$ treatment resulted in a rapid reappearance of the phosphorylated proteins of the p38 MAPK pathway as well as the adherens junction-associated proteins and filamentous actin in treated embryos. The rapid pace of the F-actin reassembly was as expected, since actin may require as little as a few seconds to repolymerize (Schmidt and Hall, 1998; Pollard et al., 2000; Gallicano, 2001). The re-establishment of the active forms of the p38 MAPK family members as well as the adherens junction-associated proteins could indicate that once the CSAID $^{\text {TM }}$ block is removed, the embryos respond immediately to re-establish a normal developmental programme. The ability of the treated embryos to recover and re-engage the normal developmental programme after CSAID ${ }^{\mathrm{TM}}$ treatment demonstrates that the treatment is not permanently debilitating.

The most intriguing finding of our study is that treatment of 2- and 4-cell-stage embryos with CSAID $^{\text {TM }}$ drugs did not result in a blockade of development or the depolymerization of actin. A 
down-regulation of MK-p and HSP-p was observed, indicating that CSAID $^{\mathrm{TM}}$ treatment is still able to inactivate the p38 MAPK pathway at these cell stages. This result suggests that the p38 MAPK may be active in these early cleavage stages but not as a primary regulator of filamentous actin.

In conclusion, p38 MAPKs regulate many cellular processes such as inflammation, cytoskeletal rearrangements, as well as cell proliferation and apoptosis in several different cellular systems (Enslen et al., 2000; Ono and Han, 2000; Kyriakis and Avruch, 2001). The principal findings of our study clearly indicate that the p38 MAPK pathway exerts a stage-specific control over actin polymerization during preimplantation development.

\section{Materials and methods}

\section{Materials}

SB203580, SB220025, SB202474 and PD098059 were purchased from Calbiochem (La Jolla, CA, U.S.A.). These compounds were prepared in DMSO to make stock concentrations of $10 \mathrm{mM}$ and were stored at $-20^{\circ} \mathrm{C}$. All embryo culture was performed in KSOMaa (Ho et al., 1995).

\section{Antisera and actin labelling}

Rabbit antisera raised against the phosphorylated human forms of phospho-MAPKAPK2, phospho-HSP25/27 and phosphop44/42 were used (New England Biolabs, Beverly, MA, U.S.A.) (Sarkar et al., 2002; Wu and Janknecht, 2002; Yu et al., 2002). The $\alpha$ - and $\beta$-catenin antisera used were mouse monoclonal antibodies raised against human $\alpha$ - and $\beta$-catenins respectively (BD Transduction Laboratories, San Diego, CA, U.S.A.). All primary antibodies were tested over a range of concentrations and were most effective at a dilution of 1:100 from the commercial stock concentration. Primary antibodies were labelled using FITC-conjugated donkey anti-rabbit secondary antisera (Jackson Immunoresearch Laboratories, West Grove, MA, U.S.A.) and FITC-conjugated donkey anti-mouse secondary antisera (Jackson Immunoresearch Laboratories) respectively. F-actin was stained using rhodamine-conjugated phalloidin and DNA was visualized using DAPI (4,6-diamidino-2-phenylindole)

\section{Super-ovulation and embryo collection}

Female CD-1 mice (Charles River, Canada), 4-5 weeks of age, were injected with 10 i.u. of PMSG (pregnant mare's serum gonadotropin) (Sigma, St. Louis, MO, U.S.A.), followed by 10 i.u. of hCG (human chorionic gonadotropin; Sigma) $48 \mathrm{~h}$ later and just before mating with CB6F1/J males. Successful mating was determined the next morning by the presence of a vaginal plug and was considered day 0.5 of development. Timing post-hCG was used to measure embryonic development and the following embryo stages and times post-hCG were used in the present study: 2-cell, $48 \mathrm{~h}$; 4-cell, $60 \mathrm{~h}$; and 8 -cell, $72 \mathrm{~h}$. Two-cell to 8-cell-stage embryos were flushed from oviducts of female mice using flushing medium I $(1.71 \mathrm{mM}$ calcium lactate, $0.25 \mathrm{mM}$ sodium pyruvate, $3 \mathrm{mg} / \mathrm{ml} \mathrm{BSA}$ and $10 \times$ Leibovitz-modified Hanks balanced salt solution, all diluted with water to $1 \times$ ) (Spindle, 1980). Animal care and treatment were according to the guidelines of the University of Western Ontario Animal Care Committee.

\section{Whole-mount indirect immunofluorescence}

Localization of the p38 MAPK signalling pathway members, catenins and actin, in preimplantation stage mouse embryos, was assessed by whole-mount indirect immunofluorescence methods combined with observation using a laser-scanning confocal microscope. For each antiserum, the experiment was applied to a minimum of three replicate embryo series, representing a minimum of 50 embryos for each treatment group, including the drug control, vehicle control and drug treatments. Negative controls were also conducted in which embryos were exposed to the same procedure in the absence of primary antibody to assess the levels of background and non-specific binding of secondary antibody. After collection, embryos were washed once with $1 \times$ PBS and then fixed in 2\% (w/v) PFA (paraformaldehyde) in PBS for $20 \mathrm{~min}$ at room temperature $\left(20^{\circ} \mathrm{C}\right)$. After fixation, embryos were washed with $1 \times$ PBS and either processed immediately for immunostaining or stored at $4{ }^{\circ} \mathrm{C}$ in $1 \times \mathrm{PBS}+0.09 \%$ sodium azide (embryo storage buffer) for up to 2 weeks.

For immunostaining, fixed embryos were permeabilized and blocked in $1 \times$ PBS $+5 \%(\mathrm{v} / \mathrm{v})$ donkey serum $+0.01 \%$ Triton $\mathrm{X}-100$ for $1 \mathrm{~h}$ at room temperature. Embryos were washed with $1 \times$ PBS and incubated with primary antibody diluted $1: 100$ in $1 \times$ PBS $+1 \%$ donkey serum $+0.005 \%$ Triton $\mathrm{X}-100$ for $1 \mathrm{~h}$ at room temperature followed by additional washes totalling $1 \mathrm{~h}$ at $37^{\circ} \mathrm{C}$. Primary antibodies were detected by exposure for $1 \mathrm{~h}$ to FITC-conjugated secondary antibodies (Jackson Immunoresearch Laboratories) diluted 1:200. Embryos were then treated with $5 \mu \mathrm{g} / \mathrm{ml}(1: 20)$ rhodamine-conjugated phalloidin and $1 \mathrm{mg} / \mathrm{ml}(1: 2000)$ DAPI for $30 \mathrm{~min}$ at $37^{\circ} \mathrm{C}$ followed by two washes for $2 \mathrm{~h}$ each at $37^{\circ} \mathrm{C}$. Embryos were mounted in Fluoro-Guard Antifade Mounting reagent (Bio-Rad Laboratories, Mississauga, ON, Canada). Fluorescence patterns were examined using a Zeiss LSM 410 (laser-scanning microscope) with an inverted Axiovert 100 microscope under $\times 40$ water Zeiss Plan Apochromat objective lens with a numerical aperture of 1.2. The microscope room is maintained at a temperature of $22^{\circ} \mathrm{C}$. The confocal microscope uses cooled photomultiplier tubes produced by Hamamatsu Photonics (Bridgewater, NJ, U.S.A.). The images were then captured and stored as TIFF files by the Zeiss LSM software package.

Influence of p38 MAPK inhibitors on development of 2- and 4-cell-stage embryos and the actin cytoskeleton

All experiments were replicated three times using embryos collected from separate groups of animals. For each experimental replicate, embryos were treated with the CSAID ${ }^{\mathrm{TM}}$ drugs SB203580 or SB220025 as well as an inactive analogue, SB202474. A group of embryos treated with vehicle only $(0.1 \%$ DMSO in KSOMaa) was included in each experimental replicate as a control. Murine embryos were flushed from timedpregnant oviducts at $48 \mathrm{~h}$ post-hCG to collect 2-cell-stage embryos. Half of the embryos were pooled, washed and divided 
into one of four treatment groups: (i) KSOMaa + 0.1\% DMSO, (ii) $\mathrm{KSOMaa}+2.0 \mu \mathrm{M}$ SB220025, (iii) $\mathrm{KSOMaa}+20 \mu \mathrm{M}$ SB220025 and (iv) KSOMaa $+20 \mu \mathrm{M}$ SB202474. Embryos were cultured for $12 \mathrm{~h}$ and then removed and fixed using $2 \%$ PFA before processing for whole-mount indirect immunofluorescence. The second half of the 2-cell embryos in each replicate was placed into KSOMaa medium for $12 \mathrm{~h}$ to allow for development to the 4-cell stage. At that time, they were washed and divided into one of the four treatment groups defined above. These embryos were cultured for $12 \mathrm{~h}$ before removal from treatment and fixation with $2 \%$ PFA. Whole-mount indirect immunofluorescence was then performed as described by Natale et al. (2004).

\section{Influence of p38 MAPK inhibitors on development of 8-cell-stage embryos and the actin cytoskeleton}

Murine embryos were flushed from timed-pregnant oviducts at the 8 -cell stage ( $72 \mathrm{~h}$ post-hCG), pooled, washed and divided equally into one of the four treatment groups: (i) KSOMaa + $0.1 \%$ DMSO, (ii) KSOMaa $+20 \mu \mathrm{M} \quad \mathrm{SB} 202474$, (iii) $\mathrm{KSOMaa}+20 \mu \mathrm{M}$ SB203580 and (iv) $\mathrm{KSOMaa}+20 \mu \mathrm{M}$ SB220025. Embryos were assessed for morphology and progression through cleavage divisions $12 \mathrm{~h}$ after treatment $(84 \mathrm{~h}$ post-hCG), at which time half of the embryos in groups 3 and 4 were removed from culture, washed and placed in fresh KSOMaa culture drops for the remainder of the experiment. Embryos in all groups were assessed again $24 \mathrm{~h}$ after treatment ( $96 \mathrm{~h}$ post-hCG) to determine progression to the blastocyst stage. Cell viability was also assayed at this time using a vital dye, Trypan Blue (Humason, 1979). After treatment, embryos were subjected to whole-mount indirect immunofluorescence assays and filamentous actin was stained using rhodamine-conjugated phalloidin. For the assessment of CSAID ${ }^{\mathrm{TM}}$ time-course effects, murine embryos were flushed from timed-pregnant oviducts at the 8 -cell stage $(72 \mathrm{~h}$ post-hCG), pooled, washed and divided equally into four treatment groups consisting of (i) $\mathrm{KSOMaa}+0.1 \% \mathrm{DMSO}$, (ii) KSOMaa + $2.0 \mu \mathrm{M} \mathrm{SB} 220025$, (iii) $\mathrm{KSOMaa}+20 \mu \mathrm{M} \mathrm{SB} 220025$ and (iv) $\mathrm{KSOMaa}+20 \mu \mathrm{M}$ SB202474 for 3, 6 and $12 \mathrm{~h}$. At each time point, a subset of embryos was removed and fixed in $2 \%$ PFA for processing by immunofluorescence analysis.

\section{MEK-1 inhibitor experiments}

Murine embryos were flushed from timed-pregnant oviducts at the 2 -cell stage ( $48 \mathrm{~h}$ post-hCG), pooled, washed and divided equally into four treatment groups consisting of (i) KSOMaa + $0.1 \%$ DMSO, (ii) KSOMaa $+1 \mu \mathrm{M}$ PD098059, (iii) KSOMaa $+10 \mu \mathrm{M}$ PD098059 and (iv) KSOMaa $+100 \mu \mathrm{M}$ PD098059. Embryos were assessed for morphology and progression through cleavage divisions at $24 \mathrm{~h}$ postdrug treatment $(72 \mathrm{~h}$ post-hCG), at which time point half of the embryos in groups 2, 3 and 4 were removed from culture, washed and placed in fresh KSOMaa culture drops for the remainder of the experiment. All embryos were then assessed again at $24 \mathrm{~h}$ postdrug treatment ( $96 \mathrm{~h}$ post-hCG) for progression to the blastocyst stage. Cell viability was also assayed at this time using a vital dye, Trypan Blue (Humason, 1979). After treatment, embryos were processed for whole-mount indirect immunofluorescence assays and filamentous actin was stained using rhodamine-conjugated phalloidin.

\section{Embryo recovery from CSAID ${ }^{\mathrm{TM}}$ treatment}

Murine embryos were flushed from timed-pregnant oviducts at the 8 -cell stage $(72 \mathrm{~h}$ post-hCG), pooled, washed and divided equally into one of the four treatments: (i) KSOMaa $+0.1 \%$ DMSO, (ii) KSOMaa $+2.0 \mu \mathrm{M}$ SB220025, (iii) KSOMaa + $20 \mu \mathrm{M}$ SB220025 and (iv) KSOMaa $+20 \mu \mathrm{M}$ SB202474. Embryos were cultured for $12 \mathrm{~h}(84 \mathrm{~h}$ post-hCG) and then washed with $1 \times$ PBS for $20 \mathrm{~min}$ and placed into drug-free KSOMaa for the remainder of the experiment to assay the recovery. Embryos were removed from culture at 3, 6, 12 and $24 \mathrm{~h}$ intervals (87, 90, 96 and $108 \mathrm{~h}$ post-hCG respectively) and fixed in $2 \%$ PFA for processing by immunofluorescence analysis.

\section{Statistical analysis}

Statistical analysis of data was performed using SigmaStat (Jandel Scientific, San Rafael, CA, U.S.A.) software package. Data showed normal distribution and one-way ANOVA was used to determine treatment effects, followed by Tukey's Multiple Comparison Test to determine the statistical significance. $P \leqslant 0.05$ was considered statistically significant.

\section{Acknowledgments}

We are indebted to Dr M. Calder, J. Andrassy, B. Fong and M. Violette for their help with embryo collection. We thank Dr T. Kennedy, Dr G.M. Kidder and Dr K. Leco (Department of Physiology and Pharmacology, The University of Western Ontario) for critically reviewing this paper before submission. We are grateful to N. Bechard for assistance with the confocal microscope. This work was supported by an operating grant from the Canadian Institute of Health Research (grant no. MOP-49449) to A.J.W., by the Department of Obstetrics and Gynaecology University of Western Ontario Graduate Research Scholarship to A.J.M.P. and by an Ontario Graduate Scholarship to D.R.N. A.J.W. is the recipient of a Premier's Research Excellence Award.

\section{References}

Badger, A.M., Bradbeer, J.N., Votta, B., Lee, J.C., Adams, J.L. and Griswold, D.E. (1996) Pharmacological profile of SB 203580, a selective inhibitor of cytokine suppressive binding protein/p38 kinase, in animal models of arthritis, bone resorption, endotoxin shock and immune function. J. Pharmacol. Exp. Ther. 279, 1453-1461

Benndorf, R., Hayess, K., Ryazantsev, S., Wieske, M., Behlke, J. and Lutsch, G. (1994) Phosphorylation and supramolecular organization of murine small heat shock protein HSP25 abolish its actin polymerization-inhibiting activity. J. Biol. Chem. 269, 20780-20784

Boulton, T.G. and Cobb, M.H. (1991) Identification of multiple extracellular signal-regulated kinases (ERKs) with antipeptide antibodies. Cell Regul. 2, 357-371 
Capco, D.G. (2001) Molecular and biochemical regulation of early mammalian development. Int. Rev. Cytol. 207, 195-235

Cirillo, P.F., Pargellis, C. and Regan, J. (2002) The non-diaryl heterocycle classes of p38 MAP kinase inhibitors. Curr. Top. Med. Chem. 2, 1021-1035

Clayton, L., Hall, A. and Johnson, M.H. (1999) A role for Rho-like GTPases in the polarisation of mouse eight-cell blastomeres. Dev. Biol. 205, 322-331

Cuenda, A., Rouse, J., Doza, Y.N., Meier, R., Cohen, P., Gallagher T.F., Young, P.R. and Lee, J.C. (1995) SB 203580 is a specific inhibitor of a MAP kinase homologue which is stimulated by cellular stresses and interleukin-1. FEBS Lett. 364, 229-233

Dalle-Donne, I., Rossi, R., Milzani, A., Di Simplicio, P. and Colombo, R. (2001) The actin cytoskeleton response to oxidants: from small heat shock protein phosphorylation to changes in the redox state of actin itself. Free Radical. Biol. Med. 31, 1624-1632

Davidson, S.M. and Morange, M. (2000) Hsp25 and the p38 MAPK pathway are involved in differentiation of cardiomyocytes. Dev. Biol. 218, 146-160

Davies, S.P., Reddy, H., Caivano, M. and Cohen, P. (2000) Specificity and mechanism of action of some commonly used protein kinase inhibitors. Biochem. J. 351, 95-105

English, J.M. and Cobb, M.H. (2002) Pharmacological inhibitors of MAPK pathways. Trends Pharmacol. Sci. 23, 40-45

Enslen, H., Brancho, D.M. and Davis, R.J. (2000) Molecular determinants that mediate selective activation of p38 MAP kinase isoforms. EMBO J. 19, 1301-1311

Fleming, T.P., Butler, L., Lei, X., Collins, J., Javed, Q., Sheth, B., Stoddart, N., Wild, A. and Hay, M. (1994) Molecular maturation of cell adhesion systems during mouse early development. Histochemistry 101, 1-7

Gallicano, G.I. (2001) Composition, regulation, and function of the cytoskeleton in mammalian eggs and embryos. Front. Biosci. 6 D1089-D1108

Gerthoffer, W.T. and Gunst, S.J. (2001) Focal adhesion and small heat shock proteins in the regulation of actin remodeling and contractility in smooth muscle. J. Appl. Physiol. 91, 963-972

Goval, J.J., Van Cauwenberge, A. and Alexandre, H. (2000) Respective roles of protein tyrosine kinases and protein kinases $\mathrm{C}$ in the upregulation of beta-catenin distribution, and compaction in mouse preimplantation embryos: a pharmacological approach. Biol. Cell 92, 513-526

Guay, J., Lambert, H., Gingras-Breton, G., Lavoie, J.N., Huot, J. and Landry, J. (1997) Regulation of actin filament dynamics by p38 map kinase-mediated phosphorylation of heat shock protein 27 . J. Cell Sci. 110, 357-368

Han, J., Lee, J.D., Bibbs, L. and Ulevitch, R.J. (1994) A MAP kinase targeted by endotoxin and hyperosmolarity in mammalian cells. Science 265, 808-811

Ho, Y., Wigglesworth, K., Eppig, J.J. and Schultz, R.M. (1995) Preimplantation development of mouse embryos in KSOM: augmentation by amino acids and analysis of gene expression. Mol. Reprod. Dev. 41, 232-238

Humason, G.L. (1979) Animal Tissue Techniques, W.H. Freeman, San Francisco, CA

Huot, J., Houle, F., Rousseau, S., Deschesnes, R.G., Shah, G.M. and Landry, J. (1998) SAPK2/p38-dependent F-actin reorganization regulates early membrane blebbing during stress-induced apoptosis. J. Cell Biol. 143, 1361-1373

Jackson, J.R., Bolognese, B., Hillegass, L., Kassis, S., Adams, J., Griswold, D.E. and Winkler, J.D. (1998) Pharmacological effects of SB 220025, a selective inhibitor of P38 mitogen-activated protein kinase, in angiogenesis and chronic inflammatory disease models. J. Pharmacol. Exp. Ther. 284, 687-692

Khurana, A. and Dey, C.S. (2003) p38 MAPK interacts with actin and modulates filament assembly during skeletal muscle differentiation. Differentiation 71, 42-50
Kidder, G.M. and McLachlin, J.R. (1985) Timing of transcription and protein synthesis underlying morphogenesis in preimplantation mouse embryos. Dev. Biol. 112, 265-275

Kyriakis, J.M. and Avruch, J. (2001) Mammalian mitogen-activated protein kinase signal transduction pathways activated by stress and inflammation. Physiol. Rev. 81, 807-869

Lavoie, J.N., Lambert, H., Hickey, E., Weber, L.A. and Landry, J. (1995) Modulation of cellular thermoresistance and actin filament stability accompanies phosphorylation-induced changes in the oligomeric structure of heat shock protein 27. Mol. Cell. Biol. 15, 505-516

Lee, J.D., Ulevitch, R.J. and Han, J. (1995) Primary structure of BMK1: a new mammalian map kinase. Biochem. Biophys. Res. Commun. 213, 715-724

Levy, J.B., Johnson, M.H., Goodall, H. and Maro, B. (1986) The timing of compaction: control of a major developmental transition in mouse early embryogenesis. J. Embryol. Exp. Morphol. 95, 213-237

McLachlin, J.R. and Kidder, G.M. (1986) Intercellular junctional coupling in preimplantation mouse embryos: effect of blocking transcription or translation. Dev. Biol. 117, 146-155

Natale, D.R., Paliga, A.J.M., Beier, F., D'Souza, S.J.A. and Watson, A.J. (2004) p38 MAPK signaling during murine preimplantation development. Dev. Biol. 268, 76-88

Ohsugi, M., Hwang, S.Y., Butz, S., Knowles, B.B., Solter, D. and Kemler, R. (1996) Expression and cell membrane localization of catenins during mouse preimplantation development. Dev. Dyn. 206, 391-402

Ohsugi, M., Larue, L., Schwarz, H. and Kemler, R. (1997) Celljunctional and cytoskeletal organization in mouse blastocysts lacking E-cadherin. Dev. Biol. 185, 261-271

Ohsugi, M., Butz, S. and Kemler, R. (1999) Beta-catenin is a major tyrosine-phosphorylated protein during mouse oocyte maturation and preimplantation development. Dev. Dyn. 216, 168-176

Ono, K. and Han, J. (2000) The p38 signal transduction pathway: activation and function. Cell. Signal. 12, 1-13

Pauken, C.M. and Capco, D.G. (1999) Regulation of cell adhesion during embryonic compaction of mammalian embryos: roles for PKC and beta-catenin. Mol. Reprod. Dev. 54, 135-144

Pauken, C.M. and Capco, D.G. (2000) The expression and stage-specific localization of protein kinase $C$ isotypes during mouse preimplantation development. Dev. Biol. 223, 411-421

Perez-Moreno, M., Jamora, C. and Fuchs, E. (2003) Sticky business: orchestrating cellular signals at adherens junctions. Cell (Cambridge, Mass.) 112, 535-548

Pollard, T.D., Blanchoin, L. and Mullins, R.D. (2000) Molecular mechanisms controlling actin filament dynamics in nonmuscle cells. Annu. Rev. Biophys. Biomol. Struct. 29, 545-576

Pouyssegur, J., Volmat, V. and Lenormand, P. (2002) Fidelity and spatio-temporal control in MAP kinase (ERKs) signalling. Biochem. Pharmacol. 64, 755-763

Pratt, H.P., Ziomek, C.A., Reeve, W.J. and Johnson, M.H. (1982) Compaction of the mouse embryo: an analysis of its components. J. Embryol. Exp. Morphol. 70, 113-132

Sarkar, D., Su, Z.Z., Lebedeva, I.V., Sauane, M., Gopalkrishnan, R.V., Valerie, K., Dent, P. and Fisher, P.B. (2002) mda-7 (IL-24) mediates selective apoptosis in human melanoma cells by inducing the coordinated overexpression of the GADD family of genes by means of p38 MAPK. Proc. Natl. Acad. Sci. U.S.A. 99, 10054-10059

Schmidt, A. and Hall, M.N. (1998) Signaling to the actin cytoskeleton. Annu. Rev. Cell Dev. Biol. 14, 305-338

Schultz, R.M. (2002) The molecular foundations of the maternal to zygotic transition in the preimplantation embryo. Hum. Reprod. Update 8, 323-331

Spindle, A. (1980) An improved culture medium for mouse blastocysts. In Vitro 16, 669-674 
Watson, A.J. (1992) The cell biology of blastocyst development. Mol. Reprod. Dev. 33, 492-504

Watson, A.J. and Barcroft, L.C. (2001) Regulation of blastocyst formation. Front. Biosci. 6, D708-D730

Watson, A.J., Kidder, G.M. and Schultz, G.A. (1992) How to make a blastocyst. Biochem. Cell Biol. 70, 849-855

Whitmarsh, A.J. and Davis, R.J. (2001) Analyzing JNK and p38 mitogen-activated protein kinase activity. Methods Enzymol. 332, 319-336

Woodgett, J.R., Avruch, J. and Kyriakis, J. (1996) The stress activated protein kinase pathway. Cancer Surv. 27, 127-138
Wu, J. and Janknecht, R. (2002) Regulation of the ETS transcription factor ER81 by the $90-\mathrm{kDa}$ ribosomal S6 kinase 1 and protein kinase A. J. Biol. Chem. 277, 42669-42679

Yu, C., Dai, Y., Dent, P. and Grant, S. (2002) Coadministration of UCN-01 with MEK1/2 inhibitors potently induces apoptosis in BCR/ABL + leukemia cells sensitive and resistant to ST1571. Cancer Biol. Ther. 1, 674-682

Zhou, G., Bao, Z.Q. and Dixon, J.E. (1995) Components of a new human protein kinase signal transduction pathway. J. Biol. Chem. 270, 12665-12669

Received 30 September 2004/24 November 2004; accepted 16 December 2004

Published as Immediate Publication 25 April 2005, DOI 10.1042/BC20040146 